\section{First CRISPR therapy dosed}

An adult with congenital blindness is the first person to receive an in vivo CRISPR-based therapy, according to the sponsors of the clinical trial: Editas Medicine and Allergan. The trial is testing whether EDIT-101 (also known as AGN-151587) can remove a point mutation in the CEP290 gene, which causes type 10 of the retinal degenerative disease Leber congenital amaurosis (LCA). The IVS26 mutation leads to a functional loss in the CEP290 protein, which causes defects in retinal photoreceptors and severe vision loss. To correct the IVS26 mutation, EDIT-101 uses a construct containing the adenovirus vector AAV5 with two guide RNAs to identify the location of the IVS26, combined with DNA encoding the Cas9 enzyme under a promoter specific to photoreceptor cells. Another gene therapy for LCA is Luxturna (voretigene neparvovec-rzyl) from Spark Therapeutics, now owned by Roche. Both Editas and Spark use an AAV vector, but whereas Editas's genome editing corrects the mutation, Spark's agent introduces a correct copy of the affected gene. Luxturna is approved for treating specifically the form caused by mutation of the retinal pigment epithelial 65 (RPE65) gene.

In the Editas trial, 18 adult and pediatric participants will receive subretinal injection in a single eye, with any vision improvements anticipated within four weeks of treatment. Also developing a therapeutic for LCA is ProQR Therapeutics. Early results from a phase $1 / 2$ trial of sepofarsen (QR-110), an RNA-based antisense oligonucleotide therapy, showed about $60 \%$ of participants improving.

Although EDIT-101 is the first trial using a CRISPR agent inside the body, Sangamo's zinc finger nuclease therapy SB-913 was the first gene therapy used in vivo, to treat patients with the inherited metabolic disorder mucopolysaccharidosis type II. Until now, clinical trials using CRISPR-based agents had been ex vivo: patients' cells are removed, edited and then returned to the body. Initial results from a trial of CRISPR Therapeutics and Vertex's CTX001 in patients with hemoglobinrelated blood disorders suggested potentially curative responses in patients with $\beta$-thalassemia and sickle cell disease.

Published online: 7 April 2020

https://doi.org/10.1038/s41587-020-0493-4

\section{Coronavirus and the race to distribute reliable diagnostics}

\author{
International teams worked at speed to make tests for the virus available in \\ record time.
}

T he medical community is rallying to develop a set of rapid and reliable molecular diagnostic tests for the new human coronavirus that appeared in China - now dubbed sudden acute respiratory syndrome coronavirus-2 (SARS-CoV-2).

This follows a breakneck effort by several research groups to identify and sequence the virus, sharing several viral genomes in open databases over recent weeks. As infections continue to mount $-74,185$ people were reported to be infected across China as of midnight local time on 18 February - and authorities seek to accurately diagnose and document infections, the need for rapid and reliable diagnostics increases. Although many clinics still lack access to a robust, accurate and sensitive SARS-CoV-2 test, impressive progress has been made in a matter of weeks. In contrast, it took almost six months to identify and to establish assays for the coronavirus responsible for the 2002-2003 SARS outbreak.

After clusters of pneumonia cases of no known cause were epidemiologically linked to a seafood and 'wet' animal wholesale food market in Wuhan, China, three groups worked tirelessly to identify the culprit. First, researchers from Shanghai, Wuhan, Beijing and Sydney used metagenomic RNA sequencing to identify the previously unknown coronavirus in a sample from a single patient who had worked at the market. This team deposited a draft genome sequence in the publicly available GenBank sequence repository on 10 January; the current version was deposited on 17 January.

Around the same time, a rapid-response team dispatched by the Chinese Center for Disease Control and Prevention reported that they had isolated and cultivated a novel coronavirus from bronchoalveolar lavage fluid of three patients and identified it as the probable source of the outbreak. Electron microscopy observations uncovered a typical coronavirus morphology; light microscopy work demonstrated it had cytopathic effects on human airway epithelial cells. Genome sequencing revealed the virus shared over $85 \%$ sequence identity with a known SARS-like coronavirus found in bats. more genome sequences in the open access database Global Initiative on Sharing All Influenza Data (GISAID). On 12 January, this group deposited three

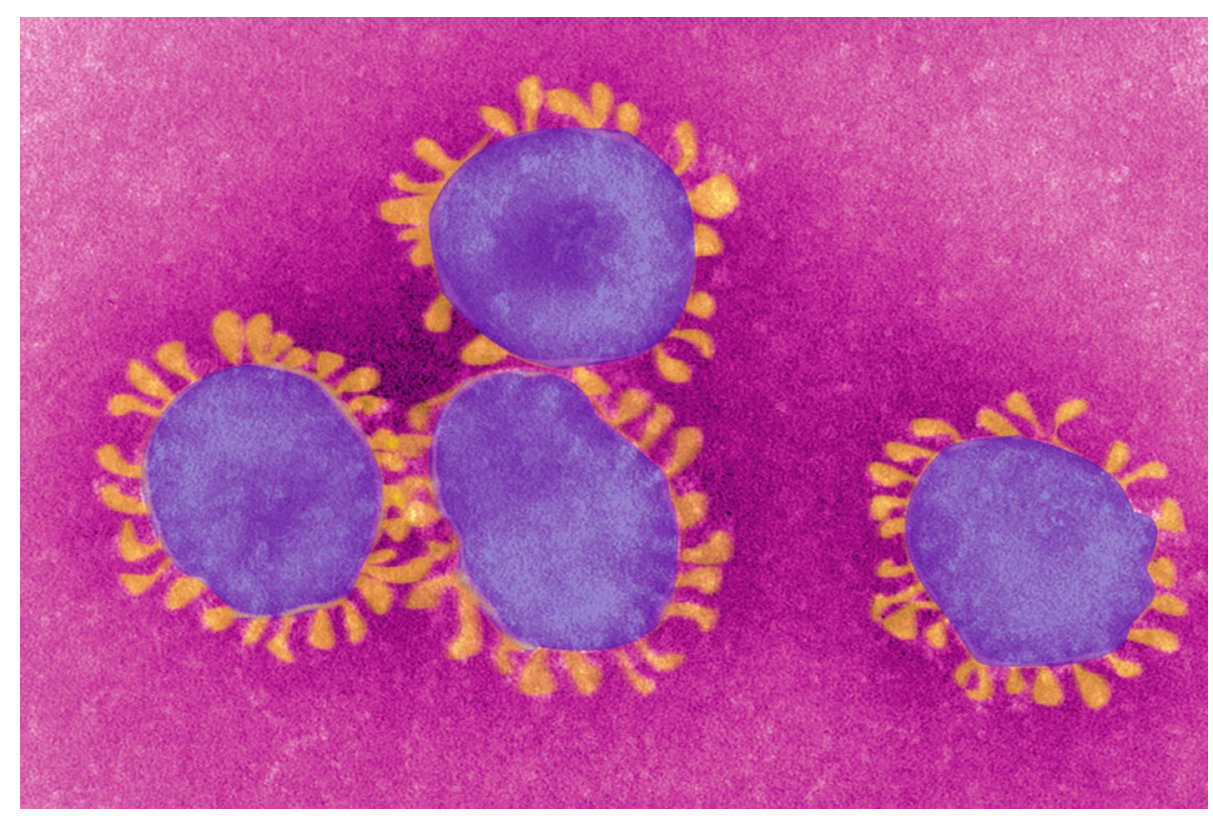

Coronaviruses are so called because the projections that encircle the capsid resemble a monarch's crown when viewed under a microscope. Credit: BSIP SA / Alamy Stock Photo 